\title{
Existence Results for the Periodic Thomas-Fermi-Dirac-von Weizsäcker Equations
}

\author{
Shaowei Chen, ${ }^{1}$ Lishan Lin, ${ }^{2}$ and Liqin Xiao ${ }^{1}$ \\ ${ }^{1}$ School of Mathematical Sciences, Huaqiao University, Quanzhou 362021, China \\ ${ }^{2}$ School of Applied Mathematical Sciences, Xiamen University of Technology, Xiamen 361024, China \\ Correspondence should be addressed to Shaowei Chen; chensw@amss.ac.cn
}

Received 18 October 2014; Accepted 23 December 2014

Academic Editor: Hagen Neidhardt

Copyright (C) 2015 Shaowei Chen et al. This is an open access article distributed under the Creative Commons Attribution License, which permits unrestricted use, distribution, and reproduction in any medium, provided the original work is properly cited.

We consider the Thomas-Fermi-Dirac-von Weizsäcker equation $-\Delta u+V(x) u+\left(u^{2} \star(1 /|x|)\right) u=\lambda|u|^{p-2} u-|u|^{q-2} u, u \in H^{1}\left(\mathbb{R}^{3}\right)$, where $\lambda>0$ is a parameter, $2<p<q<6, V \in L^{\infty}\left(\mathbb{R}^{3}\right)$ is 1-periodic in $x_{j}$, for $j=1,2,3$, and 0 is in a spectral gap of the operator $-\Delta+V$. Using a new infinite-dimensional linking theorem, we prove that, for sufficiently small $\lambda>0$, this equation has a nontrivial solution.

\section{Introduction and Statement of Results}

In this paper, we consider the following equation:

$$
\begin{aligned}
-\Delta u & +V(x) u+\left(u^{2} \star \frac{1}{|x|}\right) u \\
& =\lambda|u|^{p-2} u-|u|^{q-2} u, \quad u \in H^{1}\left(\mathbb{R}^{3}\right),
\end{aligned}
$$

where $2<p<q<6, \lambda>0$ is a parameter,

$$
u^{2} \star \frac{1}{|x|}=\int_{\mathbb{R}^{3}} \frac{u^{2}(y)}{|x-y|} d y,
$$

and $H^{1}\left(\mathbb{R}^{3}\right)$ is the standard Sobolev space with norm $\|u\|_{H^{1}\left(\mathbb{R}^{3}\right)}=\left(\int_{\mathbb{R}^{3}}\left(|\nabla u|^{2}+u^{2}\right) d x\right)^{1 / 2}$.

The potential function $V \in L^{\infty}\left(\mathbb{R}^{N}\right)$ is 1-periodic in $x_{j}$ for $j=1,2,3$. Under this assumption, $\sigma(L)$, the spectrum of the operator

$$
L=-\Delta+V: L^{2}\left(\mathbb{R}^{3}\right) \longrightarrow L^{2}\left(\mathbb{R}^{3}\right)
$$

is a purely continuous spectrum that is bounded below and consists of closed disjoint intervals ([1, Theorem XIII.100]). Thus, the complement $\mathbb{R} \backslash \sigma(L)$ consists of open intervals called spectral gaps. We assume the following: (v) $V \in L^{\infty}\left(\mathbb{R}^{N}\right)$ is 1-periodic in $x_{j}$ for $j=1,2,3$ and 0 is in a spectral gap $(-\alpha, \beta)$ of $-\Delta+V$, where $0<\alpha, \beta<$ $+\infty$.

A solution $u$ of $(1)$ is called nontrivial if $u \neq \equiv 0$. Our main result is as follows.

Theorem 1. Suppose that $2<p<q<6$ and (v) is satisfied. Then, there exists $\lambda_{0}>0$ such that, for any $0<\lambda<\lambda_{0}$, the problem (1) has a nontrivial solution.

Equation (1) arises in the study of the Thomas-FermiDirac-von Weizsäcker (TFDW) model for atoms and molecules with no external potential, where $\rho=u^{2}$ is the electron density. In [2, section VIII], Lieb studied the existence and symmetric and asymptotic properties of solutions to

$$
\begin{aligned}
- & A \Delta \psi+(V+\alpha) \psi+\left(\psi^{2} \star|x|^{-1}\right) \psi+\gamma \psi^{2 p-1}-C_{e} \psi^{5 / 3} \\
=-\mu \psi & \text { in } \mathbb{R}^{3}
\end{aligned}
$$

for various choices of $V$. Here, $A, \alpha, \gamma, C_{e}$, and $\mu$ are constants and $p>4 / 3$. The term $-C_{e} \psi^{5 / 3}$ is called the Dirac term 
with strength $C_{e}$. In [3], Le Bris considered the minimizing problem

$$
\inf _{\psi \in H^{1}\left(\mathbb{R}^{3}\right), \int_{\mathbb{R}^{3}} \psi^{2} d x=\lambda} E(\psi)
$$

with

$$
\begin{aligned}
E(\psi)= & \int_{\mathbb{R}^{3}}|\nabla \psi|^{2} d x+\int_{\mathbb{R}^{3}} V(x) \psi^{2} d x \\
& +c_{1} \int_{\mathbb{R}^{3}} \psi^{10 / 3} d x-c_{2} \int_{\mathbb{R}^{3}} \psi^{8 / 3} d x \\
& +\int_{\mathbb{R}^{3}} \int_{\mathbb{R}^{3}} \frac{\psi^{2}(x) \psi^{2}(y)}{|x-y|} d x,
\end{aligned}
$$

where $c_{1}$ and $c_{2}$ are positive constant, and $V$ is the Coulomb potential created by the atomic nuclei. Le Bris proved that there exists a $\lambda_{c}>0$, such that, for $0<\lambda<\lambda_{c}$, the problem admits a minimizer. Moreover, if $c_{2}$ is small enough, then the minimizer is unique. Using the Lagrange multiplier, it is easy to see that the minimizer of (5) is a solution to

$$
\begin{aligned}
-\Delta \psi & +V(x) \psi+\left(\psi^{2} \star|x|^{-1}\right) \psi+c_{1} \psi^{7 / 3}-c_{2} \psi^{5 / 3} \\
& =\mu \psi \quad \text { in } \mathbb{R}^{3}
\end{aligned}
$$

for some $\mu$. However, in a recent paper [4] by $\mathrm{Lu}$ and Otto, the authors proved that for sufficiently large $\lambda$, the variational problem (5) with $V=0$ does not have a minimizer. Equation (1) with $V$ a periodic function is used to describe a Hartree model for crystals (see $[5,6]$ ). Moreover, when $\lambda=0$, (1) is often referred to the Thomas-Fermi-von Weizsäcker model in the literature. One can see [7] and references therein. Finally, we should mention that the so-called Schrödinger-PoissonSlater equation

$$
-\Delta u+V(x) u+\left(u^{2} \star \frac{1}{|x|}\right) u=\lambda|u|^{p-2} u, \quad \text { in } \mathbb{R}^{3}
$$

is also related to (1) and has attracted much attention in recent years (see $[5,7-22]$ ).

The variational functional for (1) is given by

$$
\begin{aligned}
J_{\lambda}(u)= & \frac{1}{2} \int_{\mathbb{R}^{3}}\left(|\nabla u|^{2}+V(x) u^{2}\right) d x \\
& +\frac{1}{4} \int_{\mathbb{R}^{3}} \int_{\mathbb{R}^{3}} \frac{u^{2}(x) u^{2}(y)}{|x-y|} d x d y \\
& -\frac{\lambda}{p} \int_{\mathbb{R}^{3}}|u|^{p} d x+\frac{1}{q} \int_{\mathbb{R}^{3}}|u|^{q} d x, \quad u \in H^{1}\left(\mathbb{R}^{3}\right) .
\end{aligned}
$$

In other words, the critical points of $J_{\lambda}$ are solutions to (1). Hence, it is natural to use critical point theory to obtain solutions to (1). Under assumption (v), the quadratic form $\int_{\mathbb{R}^{3}}\left(|\nabla u|^{2}+V u^{2}\right) d x$ has infinite-dimensional positive and negative spaces. It can be shown that for sufficiently small $\lambda>0,-J_{\lambda}$ has a global infinite-dimensional linking geometry (for its definition, see (6.4) of Willem [23]). However, the difficulty rises when the classical infinite-dimensional linking theorem (see [23]) is used to obtain a critical point to $J_{\lambda}$. This theorem requires the functional to satisfy some upper semicontinuous assumption (see (6.3) of [23]). However, because the nonlinearity

$$
\frac{1}{4} \int_{\mathbb{R}^{3}} \int_{\mathbb{R}^{3}} \frac{u^{2}(x) u^{2}(y)}{|x-y|} d x d y+\frac{1}{q} \int_{\mathbb{R}^{3}}|u|^{q} d x-\frac{\lambda}{p} \int_{\mathbb{R}^{3}}|u|^{p} d x
$$

is neither positive definite nor negative definite in $H^{1}\left(\mathbb{R}^{3}\right)$ whenever $\lambda>0,-J_{\lambda}$ does not satisfy the upper semicontinuous assumption. To overcome this difficulty, we use a new infinite-dimensional linking theorem in $[24]$ to obtain a $(\bar{C})_{c}$ sequence (see Definition 6) for $-J_{\lambda}$. We can prove that a $(\bar{C})_{c}$ sequence for $-J_{\lambda}$ is bounded in $H^{1}\left(\mathbb{R}^{3}\right)$ if $\lambda>0$ is sufficiently small. This result is new and original. Finally, through the concentration-compactness principle and the $(\bar{C})_{c}$ sequence, a nontrivial solution to (1) is obtained. Our method can be used to study more general equation like

$$
-\Delta u+V(x) u+\left(u^{2} \star \frac{1}{|x|}\right) u=f(x, u), \quad u \in H^{1}\left(\mathbb{R}^{3}\right) .
$$

Notation. $B_{r}(a)$ denotes an open ball of radius $r$ and center a. For a Banach space $X$, we denote the dual space of $X$ by $X^{\prime}$ and denote strong and weak convergence in $X$ by $\rightarrow$ and $\rightarrow$, respectively. For $\varphi \in C^{1}(X ; \mathbb{R})$, we denote the Fréchet derivative of $\varphi$ at $u$ by $\varphi^{\prime}(u)$. The Gateaux derivative of $\varphi$ is denoted by $\left\langle\varphi^{\prime}(u), v\right\rangle$, for all $u, v \in X . L^{q}\left(\mathbb{R}^{3}\right)$ and $L_{\text {loc }}^{q}\left(\mathbb{R}^{3}\right)$ denote the standard $L^{q}$ space and the locally $q$-integrable function space, respectively $(1 \leq q \leq \infty)$. Let $\Omega$ be a domain in $\mathbb{R}^{N}(N \geq 1)$. $C_{0}^{\infty}(\Omega)$ is the space of infinitely differentiable functions with compact support in $\Omega$. We use $O(h)$ and $o(h)$ to mean $|O(h)| \leq C|h|$ and $o(h) /|h| \rightarrow 0$, respectively.

\section{Variational Setting for (1)}

Let $\mathscr{D}^{1,2}\left(\mathbb{R}^{3}\right)$ be the Hilbert space

$$
\mathscr{D}^{1,2}\left(\mathbb{R}^{3}\right)=\left\{\left.u \in L^{6}\left(\mathbb{R}^{3}\right)\left|\int_{\mathbb{R}^{3}}\right| \nabla u\right|^{2} d x<\infty\right\}
$$

with inner product

$$
(u, v)_{\mathscr{D}^{1,2}\left(\mathbb{R}^{3}\right)}=\int_{\mathbb{R}^{3}} \nabla u \nabla v d x .
$$

For $u \in H^{1}\left(\mathbb{R}^{3}\right)$, by the Lax-Milgram theorem, the equation

$$
-\Delta \phi=4 \pi u^{2} \text { in } \mathbb{R}^{3}
$$

has a unique solution $\phi_{u} \in \mathscr{D}^{1,2}\left(\mathbb{R}^{3}\right)$ (see Proposition 2.2 of [10]). And, by Theorem 2.2.1 of [25], $\phi_{u}$ can be expressed by

$$
\phi_{u}(x)=\left(u^{2} \star \frac{1}{|y|}\right)(x)=\int_{\mathbb{R}^{3}} \frac{u^{2}(y)}{|x-y|} d y, \quad x \in \mathbb{R}^{3} .
$$

The function $\phi_{u}$ has the following properties. 
Lemma 2. (i) There exists a positive constant $C$ such that, for any $u \in H^{1}\left(\mathbb{R}^{3}\right)$,

$$
\begin{aligned}
& \left\|\phi_{u}\right\|_{\mathscr{D}^{1,2}\left(\mathbb{R}^{3}\right)} \leq C\|u\|_{H^{1}\left(\mathbb{R}^{3}\right)}^{2}, \\
& \int_{\mathbb{R}^{3}}\left|\nabla \phi_{u}\right|^{2} d x=4 \pi \int_{\mathbb{R}^{3}} \phi_{u} u^{2} d x \\
& =4 \pi \int_{\mathbb{R}^{3}} \int_{\mathbb{R}^{3}} \frac{u^{2}(x) u^{2}(y)}{|x-y|} d x d y \\
& \leq C\|u\|_{L^{12 / 5}\left(\mathbb{R}^{3}\right)}^{4} \leq C\|u\|_{H^{1}\left(\mathbb{R}^{3}\right)}^{4} .
\end{aligned}
$$

(ii) For any $u \in H^{1}\left(\mathbb{R}^{3}\right), \phi_{u} \geq 0$ in $\mathbb{R}^{3}$.

Proof. The inequality

$$
4 \pi \int_{\mathbb{R}^{3}} \int_{\mathbb{R}^{3}} \frac{u^{2}(x) u^{2}(y)}{|x-y|} d x d y \leq C\|u\|_{L^{12 / 5}\left(\mathbb{R}^{3}\right)}^{4}
$$

can be derived from the the Hardy-Littlewood-Sobolev inequality (see (1.1) of [26]) by choosing $n=3, \lambda=1, p=$ $t=6 / 5$, and $f=g=u^{2}$ there. For a proof of the other properties of $\phi_{u}$ in this lemma, one can see $[8,10,12]$.

By this lemma, the functional $J_{\lambda}$ in (9) is well defined in $H^{1}\left(\mathbb{R}^{3}\right)$. And a direct computation shows that the derivative of $J_{\lambda}$ is

$$
\begin{aligned}
&\left\langle J_{\lambda}^{\prime}(u), v\right\rangle= \int_{\mathbb{R}^{3}}(\nabla u \nabla v+V(x) u v) d x \\
&+\int_{\mathbb{R}^{3}} \int_{\mathbb{R}^{3}} \frac{u(x) v(x) u^{2}(y)}{|x-y|} d x d y \\
&-\lambda \int_{\mathbb{R}^{3}}|u|^{p-2} u v d x+\int_{\mathbb{R}^{3}}|u|^{q-2} u v d x, \\
& \forall u, v \in H^{1}\left(\mathbb{R}^{3}\right) .
\end{aligned}
$$

It is easy to verify that $J_{\lambda}$ is a $C^{1}$ functional in $H^{1}\left(\mathbb{R}^{3}\right)$. Moreover, we have the following.

Lemma 3. The following statements are equivalent:

(i) $u \in H^{1}\left(\mathbb{R}^{3}\right)$ is a solution of $(1)$;

(ii) $u$ is a critical point of $J_{\lambda}$.

Under the assumption ( $\mathbf{v})$, there is a standard variational setting for the quadratic form $\int_{\mathbb{R}^{3}}\left(|\nabla u|^{2}+V(x) u^{2}\right) d x$. One can see section 6.4 of [23]. But for the convenience of the reader, we state it here.

Let $L$ be the operator defined by (3). We denote by $|L|^{1 / 2}$ the square root of the absolute value of $L$. The domain of $|L|^{1 / 2}$ is the space

$$
X:=H^{1}\left(\mathbb{R}^{3}\right)
$$

On $X$, we choose the inner product $(u, v)=\int_{\mathbb{R}^{3}}|L|^{1 / 2} u$. $|L|^{1 / 2} v d x$ and the corresponding norm $\|u\|=\sqrt{(u, u)}$. Since
0 lies in a gap of the essential spectrum of $L$, there exists an orthogonal decomposition $X=Y \oplus Z$ such that $Y$ and $Z$ are the positive and negative spaces corresponding to the spectral decomposition of $L$. Since $V$ is 1 -periodic for all variables, they are invariant under the action of $\mathbb{Z}^{3}$, that is, for any $u \in Y$ or $u \in Z$ and for any $\mathbf{k}=\left(n_{1}, n_{2}, n_{3}\right) \in \mathbb{Z}^{3}, u(\cdot-\mathbf{k})$ is also in $Y$ or $Z$. Furthermore,

$$
\begin{gathered}
\forall u \in Y, \quad \int_{\mathbb{R}^{3}}\left(|\nabla u|^{2}+V u^{2}\right) d x=(u, u)=\|u\|^{2}, \\
\forall u \in Z, \quad \int_{\mathbb{R}^{3}}\left(|\nabla u|^{2}+V u^{2}\right) d x=-(u, u)=-\|u\|^{2} .
\end{gathered}
$$

Let $Q: X \rightarrow Z, P: X \rightarrow Y$ be the orthogonal projections. By (21),

$$
\int_{\mathbb{R}^{3}}\left(|\nabla u|^{2}+V u^{2}\right) d x=\|P u\|^{2}-\|Q u\|^{2}, \quad \forall u \in X .
$$

Moreover, by $X=Y \oplus Z$, we have

$$
u=P u+Q u, \quad\|u\|^{2}=\|P u\|^{2}+\|Q u\|^{2}, \quad \forall u \in X .
$$

By (22) and (9),

$$
\begin{aligned}
-J_{\lambda}(u)= & \frac{1}{2}\|Q u\|^{2}-\frac{1}{2}\|P u\|^{2}-\frac{1}{4} \int_{\mathbb{R}^{3}} \int_{\mathbb{R}^{3}} \frac{u^{2}(x) u^{2}(y)}{|x-y|} d x d y \\
& +\frac{\lambda}{p} \int_{\mathbb{R}^{3}}|u|^{p} d x-\frac{1}{q} \int_{\mathbb{R}^{3}}|u|^{q} d x, \quad u \in X .
\end{aligned}
$$

Moreover, by (19),

$$
\begin{aligned}
\left\langle-J_{\lambda}^{\prime}(u), v\right\rangle= & (Q u, v)-(P u, v) \\
& -\int_{\mathbb{R}^{3}} \int_{\mathbb{R}^{3}} \frac{u(x) v(x) u^{2}(y)}{|x-y|} d x d y \\
& +\lambda \int_{\mathbb{R}^{3}}|u|^{p-2} u v d x \\
& -\int_{\mathbb{R}^{3}}|u|^{q-2} u v d x, \quad \forall u, v \in X .
\end{aligned}
$$

\section{A Global Linking Geometry for $-J_{\lambda}$}

Let $\left\{e_{k}\right\}$ be a total orthonormal sequence in $Y$ and

$$
\||u|\|=\max \left\{\|Q u\|, \sum_{k=1}^{\infty} \frac{1}{2^{k+1}}\left|\left(P u, e_{k}\right)\right|\right\} .
$$

For $R>r>0$ and $u_{0} \in Z$ with $\left\|u_{0}\right\|=1$, set

$$
\begin{aligned}
N= & \{u \in Z \mid\|u\|=r\}, \\
M= & \left\{u+t u_{0} \mid u \in Y, t \geq 0,\left\|u+t u_{0}\right\| \leq R\right\}, \\
\partial M= & \{u \in Y \mid\|u\| \leq R\} \\
& \cup\left\{u+t u_{0} \mid u \in Y, t>0,\left\|u+t u_{0}\right\|=R\right\} .
\end{aligned}
$$


Definition 4 . Let $\phi \in C^{1}(X, \mathbb{R})$. $\phi^{\prime}$ is called weakly sequentially continuous if $u \in X$ and $\left\{u_{n}\right\} \subset X$ are such that $u_{n} \rightarrow u$; then, for any $\varphi \in X,\left\langle\phi^{\prime}\left(u_{n}\right), \varphi\right\rangle \rightarrow\left\langle\phi^{\prime}(u), \varphi\right\rangle$.

Lemma 5. The functional $-J_{\lambda}$ satisfies the following.

(a) $-J_{\lambda}^{\prime}$ is weakly sequentially continuous and, for every $\lambda>0$,

$$
\sup _{M}\left(-J_{\lambda}\right)<+\infty
$$

(b) There exist $\delta>0, R>r>0, u_{0} \in Z$ with $\left\|u_{0}\right\|=1$ and $\lambda_{0}^{\prime}>0$ such that, for $0<\lambda<\lambda_{0}^{\prime}$,

$$
\inf _{N}\left(-J_{\lambda}\right)>\max \left\{\sup _{\partial M}\left(-J_{\lambda}\right), \sup _{\|u\| \leq \delta}\left(-J_{\lambda}(u)\right)\right\} .
$$

Proof. (a) Let $u \in X$ and $\left\{u_{n}\right\} \subset X$ be such that $u_{n} \rightarrow u$ as $n \rightarrow \infty$. It follows that

$$
\begin{array}{r}
\left(Q u_{n}, v\right) \longrightarrow(Q u, v), \quad\left(P u_{n}, v\right) \longrightarrow(P u, v), \\
n \longrightarrow \infty, \forall v \in X .
\end{array}
$$

Since $u_{n} \rightarrow u$ implies $u_{n} \rightarrow u$ in $L_{\text {loc }}^{s}\left(\mathbb{R}^{3}\right)$ for any $1 \leq s<$ 6 , we get that, for any $v \in C_{0}^{\infty}\left(\mathbb{R}^{3}\right)$, as $n \rightarrow \infty$,

$$
\begin{aligned}
& \int_{\mathbb{R}^{3}}\left|u_{n}\right|^{p-2} u_{n} v d x \rightarrow \int_{\mathbb{R}^{3}}|u|^{p-2} u v d x, \\
& \int_{\mathbb{R}^{3}}\left|u_{n}\right|^{q-2} u_{n} v d x \longrightarrow \int_{\mathbb{R}^{3}}|u|^{q-2} u v d x .
\end{aligned}
$$

By Lemma 2, we have that $\left\{\phi_{u_{n}}\right\}$ is bounded in $\mathscr{D}^{1,2}\left(\mathbb{R}^{3}\right)$. Therefore, up to a subsequence, $\phi_{u_{n}} \rightarrow \phi_{u}$ as $n \rightarrow \infty$. It follows that $\phi_{u_{n}} \rightarrow \phi_{u}$ in $L_{\text {loc }}^{s}\left(\mathbb{R}^{3}\right)$ for any $1 \leq s<6$. This yields that, for any $v \in C_{0}^{\infty}\left(\mathbb{R}^{3}\right)$, as $n \rightarrow \infty$,

$$
\int_{\mathbb{R}^{3}} \phi_{u_{n}} u_{n} v d x \longrightarrow \int_{\mathbb{R}^{3}} \phi_{u} u v d x
$$

From (25), (30), (31), and (32), we deduce that, as $n \rightarrow \infty$,

$$
\left\langle-J_{\lambda}^{\prime}\left(u_{n}\right), v\right\rangle \longrightarrow\left\langle-J_{\lambda}^{\prime}(u), v\right\rangle, \quad \forall v \in C_{0}^{\infty}\left(\mathbb{R}^{3}\right) .
$$

Hence, $-J_{\lambda}^{\prime}$ is weakly sequentially continuous. Moreover, it is easy to see that $-J_{\lambda}$ maps bounded sets into bounded sets; hence $\sup _{M}\left(-J_{\lambda}\right)<+\infty$.

(b) If $u \in Z$, then $P u=0$ and $Q u=u$. Using (17), we get from (24) that, for any $u \in Z$,

$$
\begin{aligned}
-J_{\lambda}(u) \geq & \frac{1}{2}\|u\|^{2}-\frac{1}{4} \int_{\mathbb{R}^{3}} \int_{\mathbb{R}^{3}} \frac{u^{2}(x) u^{2}(y)}{|x-y|} d x d y \\
& -\frac{1}{q} \int_{\mathbb{R}^{3}}|u|^{q} d x \\
\geq & \frac{1}{2}\|u\|^{2}-\frac{C}{16 \pi}\|u\|^{4}-\frac{C_{1}}{q}\|u\|^{q},
\end{aligned}
$$

where $C_{1}$ comes from the Sobolev inequality $\|u\|_{L^{q}\left(\mathbb{R}^{3}\right)} \leq$ $C_{1}^{1 / q}\|u\|$, for all $u \in X$. It follows that we can choose sufficiently small $r>0$ such that, for $N=\{u \in Z \mid\|u\|=r\}$,

$$
\inf _{N}\left(-J_{\lambda}\right)>\frac{1}{4} r^{2}>0 .
$$

Since $q>p$, there exists $\Lambda>0$ such that

$$
\frac{\lambda}{p}|t|^{p}-\frac{1}{q}|t|^{q}<0 \quad \text { if } 0<\lambda \leq 1,|t|>\Lambda .
$$

Let $C^{\prime}>0$ be such that

$$
\|u\|_{L^{2}\left(\mathbb{R}^{3}\right)} \leq C^{\prime}\|u\|, \quad \forall u \in X .
$$

From $q>p>2$, we deduce that there exists $0<\lambda_{0}^{\prime}<1$ such that, for any $0<\lambda \leq \lambda_{0}^{\prime}$,

$$
\sup _{|t| \leq \Lambda} t^{-2}\left(\frac{\lambda}{p}|t|^{p}-\frac{1}{q}|t|^{q}\right)<\frac{1}{4 C^{\prime 2}} .
$$

From (36) and (38), we deduce that, for any $0<\lambda \leq \lambda_{0}^{\prime}$ and $u \in X$,

$$
\begin{aligned}
-J_{\lambda}(u) & \frac{1}{2}\|Q u\|^{2}-\frac{1}{2}\|P u\|^{2}-\frac{1}{4} \int_{\mathbb{R}^{3}} \int_{\mathbb{R}^{3}} \frac{u^{2}(x) u^{2}(y)}{|x-y|} d x d y \\
& +\int_{\mathbb{R}^{3}}\left(\frac{\lambda}{p}|u|^{p}-\frac{1}{q}|u|^{q}\right) d x \\
\leq & \frac{1}{2}\|Q u\|^{2}-\frac{1}{2}\|P u\|^{2}-\frac{1}{4} \int_{\mathbb{R}^{3}} \int_{\mathbb{R}^{3}} \frac{u^{2}(x) u^{2}(y)}{|x-y|} d x d y \\
& +\int_{\{x \| u(x) \mid \leq \Lambda\}}\left(\frac{\lambda}{p}|u|^{p}-\frac{1}{q}|u|^{q}\right) d x \\
\leq & \frac{1}{2}\|Q u\|^{2}-\frac{1}{2}\|P u\|^{2}-\frac{1}{4} \int_{\mathbb{R}^{3}} \int_{\mathbb{R}^{3}} \frac{u^{2}(x) u^{2}(y)}{|x-y|} d x d y \\
& +\frac{1}{4 C^{\prime 2}} \int_{\mathbb{R}^{3}} u^{2} d x \\
\leq & \frac{1}{2}\|Q u\|^{2}-\frac{1}{2}\|P u\|^{2} \\
= & \frac{3}{4}\|Q u\|^{2}-\frac{1}{4}\|P u\|^{2}-\frac{1}{4} \int_{\mathbb{R}^{3}} \int_{\mathbb{R}^{3}} \frac{u^{2}(x) u^{2}(y)}{|x-y|} d x d y . \\
& \frac{1}{\mathbb{R}^{3}} \frac{u^{2}(x) u^{2}(y)}{|x-y|} d x d y+\frac{1}{4}\|u\|^{2}
\end{aligned}
$$

From Theorem 1.1 of [27], we deduce that there exists $C_{*}>0$ such that, for any $u \in X$,

$$
\begin{aligned}
\int_{\mathbb{R}^{3}} \int_{\mathbb{R}^{3}} \frac{u^{2}(x) u^{2}(y)}{|x-y|} d x d y \\
\quad \geq C_{*}\left(\int_{\mathbb{R}^{3}} \frac{u^{2}}{|x|^{1 / 2}(1+|\ln | x||)} d x\right)^{2} .
\end{aligned}
$$


Let $u_{0} \in Z$ be such that $\left\|u_{0}\right\|=1$. And let $u=v+t u_{0} \in$ $Y \oplus \mathbb{R} u_{0}$. Since there exists a continuous projection

$$
L^{2}\left(\mathbb{R}^{3},|x|^{-1 / 2}(1+|\ln | x||)^{-1}\right) \longrightarrow \mathbb{R} u_{0},
$$

we deduce that there exists a constant $C_{* *}>0$ such that, for any $u=v+t u_{0} \in Y \oplus \mathbb{R} u_{0}$,

$$
\left(\int_{\mathbb{R}^{3}} \frac{u^{2}}{|x|^{1 / 2}(1+|\ln | x||)} d x\right)^{2} \geq C_{* *} t^{4} .
$$

Combining (39)-(42), we deduce that there exists a constant $\kappa>0$ such that, for any $u=t u_{0}+v \in Y \oplus \mathbb{R} u_{0}$,

$$
-J_{\lambda}(u) \leq \frac{3}{4} t^{2}-\frac{1}{4}\|v\|^{2}-\kappa t^{4} .
$$

It follows that

$$
-J_{\lambda}(u) \longrightarrow-\infty, \quad \text { as }\|u\| \longrightarrow \infty, u \in Y \oplus \mathbb{R} u_{0} .
$$

Moreover, for $0<\lambda \leq \lambda_{0}^{\prime}$ and $u \in Y$, (39) implies

$$
-J_{\lambda}(u) \leq-\frac{1}{4}\|u\|^{2}-\frac{1}{4} \int_{\mathbb{R}^{3}} \int_{\mathbb{R}^{3}} \frac{u^{2}(x) u^{2}(y)}{|x-y|} d x d y \leq 0 .
$$

Together with (44), this implies that there exists $R>r$ such that

$$
\sup _{\partial M}\left(-J_{\lambda}\right) \leq 0<\inf _{N}\left(-J_{\lambda}\right) .
$$

From (39) and the definition of $\||\cdot|\|$ (see (26)), we have, for $0<\lambda \leq \lambda_{0}^{\prime}$,

$$
-J_{\lambda}(u) \leq \frac{3}{4}\|Q u\|^{2} \leq \frac{3}{4}\||u|\|^{2} .
$$

Choosing $\delta=r / \sqrt{6}$, (47) and (35) give that

$$
\sup _{\|u\| \leq \delta}\left(-J_{\lambda}(u)\right)<\inf _{N}\left(-J_{\lambda}\right) .
$$

Together with (46), this yields (29).

\section{Boundedness of $(\bar{C})_{c}$ Sequence of $-J_{\lambda}$}

Definition 6. Let $\phi \in C^{1}(X, \mathbb{R})$. A sequence $\left\{u_{n}\right\} \subset X$ is called a $(\bar{C})_{c}$ sequence for $\phi$, if

$$
\begin{aligned}
\sup _{n} \phi\left(u_{n}\right) \leq c, \quad\left(1+\left\|u_{n}\right\|\right)\left\|\phi^{\prime}\left(u_{n}\right)\right\|_{X^{\prime}} & \longrightarrow 0, \\
\text { as } n & \longrightarrow \infty .
\end{aligned}
$$

In this section, we will prove that if $\lambda>0$ is sufficiently small, then a $(\bar{C})_{c}$ sequence for $-J_{\lambda}$ is bounded in $X$.

Since $q>p>2$, there exists $D>0$ such that

$$
|t|^{q-2}-\lambda|t|^{p-2}>\left\|V_{-}\right\|_{L^{\infty}\left(\mathbb{R}^{3}\right)} \quad \text { if } 0<\lambda \leq 1,|t|>\frac{D}{2},
$$

where $V_{-}(x)=\max \{-V(x), 0\}, x \in \mathbb{R}^{3}$.
Lemma 7. Suppose that $0<\lambda \leq 1$. If $\left\{u_{n}\right\}$ is a $(\bar{C})_{c}$ sequence for $-J_{\lambda}$, then

$$
\lim _{n \rightarrow \infty} \int_{\left\{x|| u_{n}(x) \mid>D\right\}}\left|u_{n}\right|^{6} d x=0 .
$$

Proof. Let $v_{n}=\max \left\{u_{n}(x)-D / 2,0\right\}$. It is easy to verify that $v_{n} \in X$ and $\left\|v_{n}\right\| \leq\left\|u_{n}\right\|$, for all $n \in \mathbb{N}$. Together with the fact that $\left\{u_{n}\right\}$ is a $(\bar{C})_{c}$ sequence of $-J_{\lambda}$, this implies $\left\langle J^{\prime}\left(u_{n}\right), v_{n}\right\rangle=$ $o(1)$, where $o(1)$ denotes the infinitesimal depending only on $n$; that is, $o(1) \rightarrow 0$ as $n \rightarrow \infty$. Choosing $v=v_{n}$ in (19), by (50) and the fact that $\phi_{u_{n}}, u_{n}$, and $v_{n}$ are nonnegative in $\left\{x \mid u_{n}(x)>D / 2\right\}$, we have

$$
\begin{aligned}
o(1)= & \left\langle J^{\prime}\left(u_{n}\right), v_{n}\right\rangle \\
= & \int_{\mathbb{R}^{3}}\left|\nabla v_{n}\right|^{2} d x+\int_{\mathbb{R}^{3}} V(x) u_{n} v_{n} d x \\
& +\int_{\mathbb{R}^{3}} \phi_{u_{n}} \cdot u_{n} \cdot v_{n} d x \\
& +\int_{\mathbb{R}^{3}}\left(\left|u_{n}\right|^{q-2}-\lambda\left|u_{n}\right|^{p-2}\right) u_{n} v_{n} d x \\
= & \int_{\mathbb{R}^{3}}\left|\nabla v_{n}\right|^{2} d x+\int_{\mathbb{R}^{3}} V_{+} u_{n} v_{n} d x \\
& +\int_{\left\{x \mid u_{n}(x)>D / 2\right\}} \phi_{u_{n}} \cdot u_{n} \cdot v_{n} d x \\
& +\int_{\left\{x \mid u_{n}(x)>D / 2\right\}}\left(\left|u_{n}\right|^{q-2}-\lambda\left|u_{n}\right|^{p-2}\right. \\
\geq & \int_{\mathbb{R}^{3}}\left|\nabla v_{n}\right|^{2} d x \geq \widetilde{C}\left(\int_{\mathbb{R}^{3}}\left|v_{n}\right|^{6} d x\right)^{1 / 3} \\
= & \widetilde{C}\left(\int_{\left\{x \mid u_{n}(x)>D / 2\right\}}\left|v_{n}\right|^{6} d x\right)^{1 / 3},
\end{aligned}
$$

where $V_{+}=V+V_{-} \geq 0$ in $\mathbb{R}^{3}$ and $\widetilde{C}$ is the Sobolev constant. Note that $v_{n} \geq u_{n} / 2$ on $\left\{x \mid u_{n}(x)>D\right\}$. Together with (52), this implies

$$
\lim _{n \rightarrow \infty} \int_{\left\{x \mid u_{n}(x)>D\right\}}\left|u_{n}\right|^{6} d x=0 .
$$

Similarly, we can prove

$$
\lim _{n \rightarrow \infty} \int_{\left\{x \mid-u_{n}(x)>D\right\}}\left|u_{n}\right|^{6} d x=0 .
$$

The result of this lemma follows from these two limits.

Lemma 8. There exists $\lambda_{0}^{\prime \prime}>0$ such that if $0<\lambda<\lambda_{0}^{\prime \prime}$ and $\left\{u_{n}\right\}$ is a $(\bar{C})_{c}$ sequence for $-J_{\lambda}$, then $\left\{u_{n}\right\}$ is bounded in $X$.

Proof. By $\left(1+\left\|u_{n}\right\|\right)\left\|-J_{\lambda}^{\prime}\left(u_{n}\right)\right\|_{X^{\prime}} \rightarrow 0$, we have

$$
\left\langle-J_{\lambda}^{\prime}\left(u_{n}\right), Q u_{n}\right\rangle=o(1), \quad\left\langle-J_{\lambda}^{\prime}\left(u_{n}\right), P u_{n}\right\rangle=o(1) .
$$


6

Advances in Mathematical Physics

Choosing $v=Q u_{n}$ and $v=P u_{n}$ in (25), (55) implies that

$$
\begin{aligned}
\left\|Q u_{k}\right\|^{2}= & \int_{\mathbb{R}^{3}} u_{n} \phi_{u_{n}} \cdot Q u_{n} d x \\
& -\int_{\mathbb{R}^{3}}\left(\lambda\left|u_{n}\right|^{p-2} u_{n}-\left|u_{n}\right|^{q-2} u_{n}\right) \cdot Q u_{n} d x+o(1), \\
\left\|P u_{n}\right\|^{2}= & -\int_{\mathbb{R}^{3}} u_{n} \phi_{u_{n}} \cdot P u_{n} d x \\
& +\int_{\mathbb{R}^{3}}\left(\lambda\left|u_{n}\right|^{p-2} u_{n}-\left|u_{n}\right|^{q-2} u_{n}\right) \cdot P u_{n} d x+o(1) .
\end{aligned}
$$

By these two equalities and $\left\|u_{n}\right\|^{2}=\left\|P u_{n}\right\|^{2}+\left\|Q u_{n}\right\|^{2}$ (see (23)), we obtain

$$
\begin{array}{r}
\left\|u_{n}\right\|^{2}=\int_{\mathbb{R}^{3}} u_{n} \cdot \phi_{u_{n}} \cdot\left(Q u_{n}-P u_{n}\right) d x \\
-\int_{\mathbb{R}^{3}}\left(\lambda\left|u_{n}\right|^{p-2} u_{n}-\left|u_{n}\right|^{q-2} u_{n}\right) \\
\cdot\left(Q u_{n}-P u_{n}\right) d x+o(1) .
\end{array}
$$

Let

$$
\chi_{n, 1}(x)= \begin{cases}1, & \text { if }\left|u_{n}(x)\right| \leq D \\ 0, & \text { if }\left|u_{n}(x)\right|>D\end{cases}
$$

and $\chi_{n, 2}=1-\chi_{n, 1}$, where $D$ comes from (50). Then, $u_{n}^{2}=$ $\left(u_{n} \chi_{n, 1}\right)^{2}+\left(u_{n} \chi_{n, 2}\right)^{2}$ and

$$
\phi_{u_{n}}=\phi_{u_{n} \chi_{n, 1}}+\phi_{u_{n} \chi_{n, 2}}
$$

Since $\phi_{u_{n} \chi_{n, 1}}$ is a solution of the equation

$$
-\Delta \phi=4 \pi\left(u_{n} \chi_{n, 1}\right)^{2} \quad \text { in } \mathbb{R}^{3}
$$

by $\left(u_{n} \chi_{n, 1}\right)^{2} \leq D^{2}$ in $\mathbb{R}^{3}$ and the standard elliptic estimates (see, [28, Theorem 8.17]), we get that there exists a positive constant $C_{2}$ that is independent of $n$ and $y \in \mathbb{R}^{3}$, such that, for any $y \in \mathbb{R}^{3}$,

$$
\begin{aligned}
\left\|\phi_{u_{n} \chi_{n, 1}}\right\|_{L^{\infty}\left(B_{1}(y)\right)} & \leq C_{2}\left(\int_{B_{2}(y)}\left|u_{n} \chi_{n, 1}\right|^{4} d x\right)^{1 / 2} \\
& \leq C_{2} D^{2}\left(\int_{B_{2}(y)} d x\right)^{1 / 2} .
\end{aligned}
$$

Let $C_{3}=\left(\int_{B_{2}(0)} d x\right)^{1 / 2}$. By $(61)$,

$$
\left\|\phi_{u_{n} \chi_{n, 1}}\right\|_{L^{\infty}\left(\mathbb{R}^{3}\right)} \leq C_{2} C_{3} D^{2} .
$$

Together with $\phi_{u_{n} \chi_{n, 1}} \geq 0$ in $\mathbb{R}^{3}$ (see Lemma 2(ii)), this implies

$$
0 \leq \phi_{u_{n} \chi_{n, 1}} \leq C_{2} C_{3} D^{2} \text { in } \mathbb{R}^{3} .
$$

By (57), (59), and (63), we have

$$
\begin{aligned}
& \left\|u_{n}\right\|^{2}=\int_{\mathbb{R}^{3}} u_{n} \cdot \phi_{u_{n} \chi_{n, 1}} \cdot\left(Q u_{n}-P u_{n}\right) d x \\
& +\int_{\mathbb{R}^{3}} u_{n} \cdot \phi_{u_{n} \chi_{n, 2}} \cdot\left(Q u_{n}-P u_{n}\right) d x \\
& -\int_{\left\{x|| u_{n}(x) \mid \leq D\right\}}\left(\lambda\left|u_{n}\right|^{p-2} u_{n}-\left|u_{n}\right|^{q-2} u_{n}\right) \\
& \cdot\left(Q u_{n}-P u_{n}\right) d x \\
& -\int_{\left\{x|| u_{n}(x) \mid>D\right\}}\left(\lambda\left|u_{n}\right|^{p-2} u_{n}-\left|u_{n}\right|^{q-2} u_{n}\right) \\
& \cdot\left(Q u_{n}-P u_{n}\right) d x+o(1) \\
& \leq\left\|\phi_{u_{n} \chi_{n, 1}} u_{n}\right\|_{L^{2}\left(\mathbb{R}^{3}\right)}\left\|Q u_{n}-P u_{n}\right\|_{L^{2}\left(\mathbb{R}^{3}\right)} \\
& +\int_{\mathbb{R}^{3}} u_{n} \cdot \phi_{u_{n} \chi_{n, 2}} \cdot\left(Q u_{n}-P u_{n}\right) d x \\
& +\left(\int_{\left\{x|| u_{n}(x) \mid \leq D\right\}}\left(\lambda\left|u_{n}\right|^{p-2} u_{n}-\left|u_{n}\right|^{q-2} u_{n}\right)^{2} d x\right)^{1 / 2} \\
& \cdot\left\|Q u_{n}-P u_{n}\right\|_{L^{2}\left(\mathbb{R}^{3}\right)} \\
& +\lambda\left(\int_{\left\{x|| u_{n}(x) \mid>D\right\}}\left|u_{n}\right|^{p} d x\right)^{(p-1) / p} \\
& \cdot\left\|Q u_{n}-P u_{n}\right\|_{L^{p}\left(\mathbb{R}^{3}\right)} \\
& +\left(\int_{\left\{x|| u_{n}(x) \mid>D\right\}}\left|u_{n}\right|^{q} d x\right)^{(q-1) / q} \\
& \text { - }\left\|Q u_{n}-P u_{n}\right\|_{L^{q}\left(\mathbb{R}^{3}\right)} \text {. }
\end{aligned}
$$

Since $\left\|Q u_{n}-P u_{n}\right\|_{L^{2}\left(\mathbb{R}^{3}\right)} \leq 2 C^{\prime}\left\|u_{n}\right\|\left(C^{\prime}\right.$ is the constant coming from (37)), $\left\|Q u_{n}-P u_{n}\right\|_{L^{p}\left(\mathbb{R}^{3}\right)} \leq C^{\prime \prime}\left\|u_{n}\right\|$, and $\left\|Q u_{n}-P u_{n}\right\|_{L^{q}\left(\mathbb{R}^{3}\right)} \leq C^{\prime \prime}\left\|u_{n}\right\|$, where $C^{\prime \prime}$ is a positive constant independent of $n$ and $\lambda$, we have

$$
\begin{aligned}
\left\|u_{n}\right\|^{2} \leq & 2 C^{\prime}\left\|u_{n} \phi_{u_{n} \chi_{n, 1}}\right\|_{L^{2}\left(\mathbb{R}^{3}\right)}\left\|u_{n}\right\| \\
& +\int_{\mathbb{R}^{3}} u_{n} \cdot \phi_{u_{n} \chi_{n, 2}} \cdot\left(Q u_{n}-P u_{n}\right) d x \\
& +2 C^{\prime}\left(\int _ { \{ x | | u _ { n } ( x ) | \leq D \} } \left(\lambda\left|u_{n}\right|^{p-2} u_{n}\right.\right. \\
& +\lambda C^{\prime \prime}\left(\int_{\left\{x|| u_{n}(x) \mid>D\right\}}\left|u_{n}\right|^{p} d x\right)^{(p-1) / p}\left\|u_{n}\right\| \\
& \left.\left.+C^{\prime \prime}\left(\int_{\left\{x \| u_{n}(x) \mid>D\right\}}\left|u_{n}\right|^{q} d x\right)^{(q-1) / q} \| u_{n}\right)^{2} d x\right)^{1 / 2}\left\|u_{n}\right\|
\end{aligned}
$$


By Lemma 7, we deduce that $\int_{\left\{x|| u_{n}(x) \mid>D\right\}}\left|u_{n}\right|^{p} d x=o(1)$ and $\int_{\left\{x|| u_{n}(x) \mid>D\right\}}\left|u_{n}\right|^{q} d x=o(1)$ if $\lambda \leq 1$. Together with (63), this implies that, for $0<\lambda \leq 1$,

$$
\begin{aligned}
&\left\|u_{n}\right\|^{2} \leq 2 C^{\prime} D\left(C_{2} C_{3}\right)^{1 / 2}\left\|u_{n} \phi_{u_{n} \chi_{n, 1}}^{1 / 2}\right\|_{L^{2}\left(\mathbb{R}^{3}\right)}\left\|u_{n}\right\| \\
&+\int_{\mathbb{R}^{3}} u_{n} \cdot \phi_{u_{n} \chi_{n, 2}} \cdot\left(Q u_{n}-P u_{n}\right) d x \\
&+2 C^{\prime}\left(\int _ { \{ x | | u _ { n } ( x ) | \leq D \} } \left(\lambda\left|u_{n}\right|^{p-2} u_{n}\right.\right. \\
&\left.\left.-\left|u_{n}\right|^{q-2} u_{n}\right)^{2} d x\right)^{1 / 2}\left\|u_{n}\right\|
\end{aligned}
$$$$
+o(1) \cdot\left\|u_{n}\right\|
$$

Using the Hardy-Littlewood-Sobolev inequality (see, (1.1) of [26]), we get that

$$
\begin{aligned}
\int_{\mathbb{R}^{3}} & u_{n} \cdot \phi_{u_{n} \chi_{n, 2}} \cdot\left(Q u_{n}-P u_{n}\right) d x \\
= & \int_{\mathbb{R}^{3}} \int_{\mathbb{R}^{3}} \frac{\left(u_{n} \chi_{n, 2}(y)\right)^{2} \cdot u_{n}(x) \cdot\left(Q u_{n}-P u_{n}\right)(x)}{|x-y|} d x d y \\
\leq & C_{H L S}\left\|\left(u_{n} \chi_{n, 2}\right)^{2}\right\|_{L^{6 / 5}\left(\mathbb{R}^{3}\right)}\left\|u_{n} \cdot\left(Q u_{n}-P u_{n}\right)\right\|_{L^{6 / 5}\left(\mathbb{R}^{3}\right)} \\
\leq & C_{H L S}\left\|\left(u_{n} \chi_{n, 2}\right)^{2}\right\|_{L^{6 / 5}\left(\mathbb{R}^{3}\right)}\left\|u_{n}\right\|_{L^{12 / 5}\left(\mathbb{R}^{3}\right)} \\
& \cdot\left\|Q u_{n}-P u_{n}\right\|_{L^{12 / 5}\left(\mathbb{R}^{3}\right)} \\
\leq & C^{\prime \prime \prime}\left(\int_{\left\{x \| u_{n}(x) \mid>D\right\}}\left|u_{n}\right|^{12 / 5} d x\right)^{5 / 6}\left\|u_{n}\right\|^{2},
\end{aligned}
$$

where $C_{H L S}$ and $C^{\prime \prime \prime}$ are positive constants independent of $n$ and $\lambda$. By Lemma 7, we have

$$
\int_{\left\{x|| u_{n}(x) \mid>D\right\}}\left|u_{n}\right|^{12 / 5} d x=o(1) \quad \text { if } 0<\lambda \leq 1 .
$$

Combining (68) and (67) with (66) yields that, for $0<\lambda \leq 1$,

$$
\begin{aligned}
\left\|u_{n}\right\|^{2} \leq & 2 C^{\prime} D\left(C_{2} C_{3}\right)^{1 / 2}\left\|u_{n} \phi_{u_{n} \chi_{n, 1}}^{1 / 2}\right\|_{L^{2}\left(\mathbb{R}^{3}\right)}\left\|u_{n}\right\| \\
& +o(1) \cdot\left\|u_{n}\right\|^{2} \\
& +2 C^{\prime}\left(\int _ { \{ x | | u _ { n } ( x ) | \leq D \} } \left(\lambda\left|u_{n}\right|^{p-2} u_{n}\right.\right. \\
& \left.\left.-\left|u_{n}\right|^{q-2} u_{n}\right)^{2} d x\right)^{1 / 2}\left\|u_{n}\right\|
\end{aligned}
$$$$
+o(1) \cdot\left\|u_{n}\right\|
$$

Let $0<\epsilon<D$. Then, for $0<\lambda \leq 1$,

$$
\begin{gathered}
\left(\int_{\left\{x|| u_{n}(x) \mid \leq \epsilon\right\}}\left(\lambda\left|u_{n}\right|^{p-2} u_{n}-\left|u_{n}\right|^{q-2} u_{n}\right)^{2} d x\right)^{1 / 2} \\
\quad \leq\left(\int_{\left\{x \| u_{n}(x) \mid \leq \epsilon\right\}}\left(\left|u_{n}\right|^{p-1}+\left|u_{n}\right|^{q-1}\right)^{2} d x\right)^{1 / 2} \\
\quad \leq 2\left(\int_{\left\{x|| u_{n}(x) \mid \leq \epsilon\right\}}\left(\left|u_{n}\right|^{2 p-2}+\left|u_{n}\right|^{2 q-2}\right) d x\right)^{1 / 2} \\
\quad \leq 4\left(\epsilon^{p-2}+\epsilon^{q-2}\right)\left(\int_{\left\{x \| u_{n}(x) \mid \leq \epsilon\right\}}\left|u_{n}\right|^{2} d x\right)^{1 / 2} \\
\leq 4 C^{\prime}\left(\epsilon^{p-2}+\epsilon^{q-2}\right)\left\|u_{n}\right\| .
\end{gathered}
$$

Combining (70) with (69) yields that, for $0<\lambda \leq 1$,

$$
\begin{aligned}
\left\|u_{n}\right\|^{2} \leq & 2 C^{\prime} D\left(C_{2} C_{3}\right)^{1 / 2}\left\|u_{n} \phi_{u_{n} \chi_{n, 1}}^{1 / 2}\right\|_{L^{2}\left(\mathbb{R}^{3}\right)}\left\|u_{n}\right\| \\
& +o(1) \cdot\left\|u_{n}\right\|^{2}+4 C^{\prime 2}\left(\epsilon^{p-2}+\epsilon^{q-2}\right)\left\|u_{n}\right\|^{2} \\
& +2 C^{\prime}\left(\int _ { \{ x | \epsilon < | u _ { n } ( x ) | \leq D \} } \left(\lambda\left|u_{n}\right|^{p-2} u_{n}\right.\right. \\
& \left.\left.-\left|u_{n}\right|^{q-2} u_{n}\right)^{2} d x\right)^{1 / 2}\left\|u_{n}\right\| \\
& +o(1) \cdot\left\|u_{n}\right\| .
\end{aligned}
$$

Let $\epsilon_{0} \in\left(0, \min \left\{D, D^{1 /(q-p)}, 1\right\}\right)$ be such that $4 C^{\prime 2}\left(\epsilon_{0}^{p-2}+\right.$ $\left.\epsilon_{0}^{q-2}\right)<1 / 2$. Then, (71) implies that

$$
\begin{aligned}
\left\|u_{n}\right\|^{2} \leq & 4 C^{\prime} D\left(C_{2} C_{3}\right)^{1 / 2}\left\|u_{n} \phi_{u_{n} \chi_{n, 1}}^{1 / 2}\right\|_{L^{2}\left(\mathbb{R}^{3}\right)}\left\|u_{n}\right\| \\
& +o(1) \cdot\left\|u_{n}\right\|^{2}+o(1) \cdot\left\|u_{n}\right\| \\
& +4 C^{\prime}\left(\int _ { \{ x | \epsilon _ { 0 } < | u _ { n } ( x ) | \leq D \} } \left(\lambda\left|u_{n}\right|^{p-2} u_{n}\right.\right. \\
& \left.\left.-\left|u_{n}\right|^{q-2} u_{n}\right)^{2} d x\right)^{1 / 2}\left\|u_{n}\right\| .
\end{aligned}
$$

$\operatorname{From~sup~}_{n}\left(-J_{\lambda}\left(u_{n}\right)\right) \leq c$ and $\left(1+\left\|u_{n}\right\|\right)\left\|-J_{\lambda}^{\prime}\left(u_{n}\right)\right\|_{X^{\prime}} \rightarrow 0$, we obtain

$$
\begin{aligned}
o(1)+c \geq & -J_{\lambda}\left(u_{n}\right)+\frac{1}{2}\left\langle J_{\lambda}^{\prime}\left(u_{n}\right), u_{n}\right\rangle \\
= & \frac{1}{4} \int_{\mathbb{R}^{3}} u_{n}^{2} \phi_{u_{n}} d x \\
& -\int_{\mathbb{R}^{3}}\left(\lambda\left(\frac{1}{2}-\frac{1}{p}\right)\left|u_{n}\right|^{p}-\left(\frac{1}{2}-\frac{1}{q}\right)\left|u_{n}\right|^{q}\right) d x .
\end{aligned}
$$


It follows that

$$
\begin{aligned}
& \frac{1}{4} \int_{\mathbb{R}^{3}} u_{n}^{2} \phi_{u_{n}} d x \\
& +\int_{\left\{x|| u_{n}(x) \mid>\lambda^{1 /(q-p)\}}\right.}\left(\left(\frac{1}{2}-\frac{1}{q}\right)\left|u_{n}(x)\right|^{q}\right. \\
& \left.-\lambda\left(\frac{1}{2}-\frac{1}{p}\right)\left|u_{n}\right|^{p}\right) d x \\
& \leq c+\int_{\left\{x \| u_{n}(x) \mid \leq \lambda^{1 /(q-p)\}}\right.}\left(\lambda\left(\frac{1}{2}-\frac{1}{p}\right)\left|u_{n}\right|^{p}\right. \\
& +o(1) \\
& \leq c+C_{4} \lambda^{(q-2) /(q-p)} \int_{\mathbb{R}^{3}}\left|u_{n}\right|^{2} d x+o(1),
\end{aligned}
$$

where $C_{4}$ is a positive constant depending only on $p$ and $q$. Because $(1 / 2-1 / q)|t|^{q}-\lambda(1 / 2-1 / p)|t|^{p}>0$ if $|t| \geq \lambda^{1 /(q-p)}$, we get that, for $0<\lambda \leq \epsilon_{0}^{q-p}$,

$$
\begin{aligned}
& \int_{\left\{x|D \geq| u_{n}(x) \mid>\epsilon_{0}\right\}}\left(\left(\frac{1}{2}-\frac{1}{q}\right)\left|u_{n}\right|^{q}-\lambda\left(\frac{1}{2}-\frac{1}{p}\right)\left|u_{n}\right|^{p}\right) d x \\
& \leq \int_{\left\{x|| u_{n}(x) \mid>\lambda^{1 /(q-p)}\right\}}\left(\left(\frac{1}{2}-\frac{1}{q}\right)\left|u_{n}\right|^{q}-\lambda\left(\frac{1}{2}-\frac{1}{p}\right)\left|u_{n}\right|^{p}\right) d x .
\end{aligned}
$$

Then, by (74) and $\int_{\mathbb{R}^{3}} u_{n}^{2} \phi_{u_{n}} d x \geq \int_{\mathbb{R}^{3}} u_{n}^{2} \phi_{u_{n} \chi_{n, 1}} d x=$ $\left\|u_{n} \phi_{u_{n} \chi_{n, 1}}^{1 / 2}\right\|_{L^{2}\left(\mathbb{R}^{3}\right)}^{2}$, we get that, for $0<\lambda \leq \epsilon_{0}^{q-p}$,

$$
\begin{aligned}
& \frac{1}{4}\left\|u_{n} \phi_{u_{n} \chi_{n, 1}}^{1 / 2}\right\|_{L^{2}\left(\mathbb{R}^{3}\right)}^{2} \\
& +\int_{\left\{x|D \geq| u_{n}(x) \mid>\epsilon_{0}\right\}}\left(\left(\frac{1}{2}-\frac{1}{q}\right)\left|u_{n}\right|^{q}\right. \\
& \left.-\lambda\left(\frac{1}{2}-\frac{1}{p}\right)\left|u_{n}\right|^{p}\right) d x \\
& \leq c+C_{4} \lambda^{(q-2) /(q-p)} \int_{\mathbb{R}^{3}}\left|u_{n}\right|^{2} d x+o(1) \\
& \leq c+C^{\prime 2} C_{4} \lambda^{(q-2) /(q-p)}\left\|u_{n}\right\|^{2}+o(1) .
\end{aligned}
$$

It is easy to verify that there exists a positive constant $C_{5}$ depending only on $p, q, D$, and $\epsilon_{0}$ such that, if $0<\lambda \leq \epsilon_{0}^{q-p} / 2$ and $\epsilon_{0} \leq|t| \leq D$, then

$$
C_{5}\left(\lambda|t|^{p-2} t-|t|^{q-2} t\right)^{2} \leq\left(\frac{1}{2}-\frac{1}{q}\right)|t|^{q}-\lambda\left(\frac{1}{2}-\frac{1}{p}\right)|t|^{p} .
$$

Together with (76), this yields that, for $0<\lambda \leq \epsilon_{0}^{q-p} / 2$,

$$
\begin{aligned}
& \frac{1}{4}\left\|u_{n} \phi_{u_{n} \chi_{n, 1}}^{1 / 2}\right\|_{L^{2}\left(\mathbb{R}^{3}\right)}^{2} \\
& +C_{5} \int_{\left\{x|D \geq| u_{n}(x) \mid>\epsilon_{0}\right\}}\left(\lambda\left|u_{n}\right|^{p-2} u_{n}-\left|u_{n}\right|^{q-2} u_{n}\right)^{2} d x \\
& \leq c+C^{\prime 2} C_{4} \lambda^{(q-2) /(q-p)}\left\|u_{n}\right\|^{2}+o(1) .
\end{aligned}
$$

Combining (78) with (72), we deduce that there exists $\lambda_{0}^{\prime \prime} \epsilon$ $\left(0, \epsilon_{0}^{q-p} / 2\right)$ that is small enough such that $\left\{\left\|u_{n}\right\|\right\}$ is bounded if $0<\lambda<\lambda_{0}^{\prime \prime}$.

\section{Proof of Theorem 1}

Proof of Theorem 1. Let $\lambda_{0}=\min \left\{\lambda_{0}^{\prime}, \lambda_{0}^{\prime \prime}\right\}$, where $\lambda_{0}^{\prime}$ and $\lambda_{0}^{\prime \prime}$ are the constants in Lemmas 5 and 8 , respectively. By Lemmas 5 and 8 , and Theorem 1.3 of [24], we deduce that, for any $0<$ $\lambda<\lambda_{0}$, there exists a bounded $(\bar{C})_{c}$ sequence $\left\{u_{n}\right\}$ for $-J_{\lambda}$ with $\inf _{n}\left\|\left|u_{n}\right|\right\|>0$. Up to a subsequence, either

(a) $\lim _{n \rightarrow \infty} \sup _{y \in \mathbb{R}^{3}} \int_{B_{1}(y)}\left|u_{n}\right|^{2} d x=0$, or

(b) there exist $\varrho>0$ and $a_{n} \in \mathbb{Z}^{3}$ such that $\int_{B_{1}\left(a_{n}\right)}\left|u_{n}\right|^{2} d x \geq \varrho$.

If (a) occurs, the Lions lemma (see [23, Theorem 1.21]) implies that $u_{n}$ satisfies $u_{n} \rightarrow 0$ in $L^{s}\left(\mathbb{R}^{3}\right)$ for any $2<s<6$. It follows that

$$
\begin{aligned}
& \int_{\mathbb{R}^{3}}\left(\lambda\left|u_{n}\right|^{p-2} u_{n}-\left|u_{n}\right|^{q-2} u_{n}\right) \cdot Q u_{n} d x \longrightarrow 0, \\
& \int_{\mathbb{R}^{3}}\left(\lambda\left|u_{n}\right|^{p-2} u_{n}-\left|u_{n}\right|^{q-2} u_{n}\right) \cdot P u_{n} d x \longrightarrow 0 .
\end{aligned}
$$

As (67), we have

$$
\begin{aligned}
& \int_{\mathbb{R}^{3}} u_{n} \cdot \phi_{u_{n}} \cdot\left(Q u_{n}-P u_{n}\right) d x \\
& \quad=\int_{\mathbb{R}^{3}} \int_{\mathbb{R}^{3}} \frac{u_{n}^{2}(y) \cdot u_{n}(x) \cdot\left(Q u_{n}-P u_{n}\right)(x)}{|x-y|} d x d y \\
& \leq C_{H L S}\left\|u_{n}^{2}\right\|_{L^{6 / 5}\left(\mathbb{R}^{3}\right)}\left\|u_{n} \cdot\left(Q u_{n}-P u_{n}\right)\right\|_{L^{6 / 5}\left(\mathbb{R}^{3}\right)} \\
& \leq C_{H L S}\left\|u_{n}^{2}\right\|_{L^{6 / 5}\left(\mathbb{R}^{3}\right)}\left\|u_{n}\right\|_{L^{12 / 5}\left(\mathbb{R}^{3}\right)}\left\|Q u_{n}-P u_{n}\right\|_{L^{12 / 5}\left(\mathbb{R}^{3}\right)} \\
& \quad=C_{H L S}\left\|u_{n}\right\|_{L^{12 / 5}\left(\mathbb{R}^{3}\right)}^{3}\left\|Q u_{n}-P u_{n}\right\|_{L^{12 / 5}\left(\mathbb{R}^{3}\right)} \longrightarrow 0, \quad n \longrightarrow \infty .
\end{aligned}
$$

Combining (79) and (80) with (57) yields $\left\|u_{n}\right\| \rightarrow 0$. This contradicts $\inf _{n}\left\|\left|u_{n}\right|\right\|>0$. Therefore, case (a) cannot occur. As case (b) therefore occurs, $w_{n}=u_{n}\left(\cdot+a_{n}\right)$ satisfies $w_{n} \rightarrow$ $u_{0} \neq 0$. From $\left(1+\left\|w_{n}\right\|\right)\left\|-J_{\lambda}^{\prime}\left(w_{n}\right)\right\|_{X^{\prime}}=\left(1+\left\|u_{n}\right\|\right)$ $\left\|-J_{\lambda}^{\prime}\left(u_{n}\right)\right\|_{X^{\prime}} \rightarrow 0$ and the weakly sequential continuity of $-J_{\lambda}^{\prime}$, we have that $-J_{\lambda}^{\prime}\left(u_{0}\right)=0$. Therefore, $u_{0}$ is a nontrivial solution of (1). This completes the proof. 


\section{Conflict of Interests}

The authors declare that there is no conflict of interests regarding the publication of this paper.

\section{Acknowledgments}

The authors would like to thank the anonymous referees for their comments and suggestions on the paper. Shaowei Chen was supported by Science Foundation of Huaqiao University and Promotion Program for Young and Middle-Aged Teacher in Science and Technology Research of Huaqiao University (ZQN-PY119).

\section{References}

[1] M. Reed and B. Simon, Methods of Modern Mathematical Physics, IV: Analysis of Operators, Academic Press, New York, NY, USA, 1978.

[2] E. H. Lieb, "Thomas-Fermi and related theories of atoms and molecules," Reviews of Modern Physics, vol. 53, no. 4, pp. 603641, 1981.

[3] C. Le Bris, "Some results on the Thomas-Fermi-Dirac-von Weizsäcker model," Differential and Integral Equations, vol. 6, no. 2, pp. 337-353, 1993.

[4] J. Lu and F. Otto, "Nonexistence of a minimizer for ThomasFermi-Dirac-von Weizsäcker model," Communications on Pure and Applied Mathematics, vol. 67, no. 10, pp. 1605-1617, 2014.

[5] I. Catto, C. Le Bris, and P.-L. Lions, "On some periodic Hartreetype models for crystals," Annales de l'Institut Henri Poincare (C) Non Linear Analysis, vol. 19, no. 2, pp. 143-190, 2002.

[6] C. Le Bris and P.-L. Lions, "From atoms to crystals: a mathematical journey," Bulletin of the American Mathematical Society. New Series, vol. 42, no. 3, pp. 291-363, 2005.

[7] R. Benguria, H. Brézis, and E. H. Lieb, "The Thomas-Fermi-von Weizsäcker theory of atoms and molecules," Communications in Mathematical Physics, vol. 79, no. 2, pp. 167-180, 1981.

[8] C. O. Alves, M. A. Souto, and S. H. Soares, "SchrödingerPoisson equations without Ambrosetti-Rabinowitz condition," Journal of Mathematical Analysis and Applications, vol. 377, no. 2, pp. 584-592, 2011.

[9] S. Chen and C. Wang, "Existence of multiple nontrivial solutions for a Schrödinger-Poisson system," Journal of Mathematical Analysis and Applications, vol. 411, no. 2, pp. 787-793, 2014.

[10] T. D'Aprile and D. Mugnai, "Solitary waves for nonlinear KleinGordon-MAXwell and Schrödinger-MAXwell equations," Proceedings of the Royal Society of Edinburgh Section A, vol. 134, no. 5, pp. 893-906, 2004.

[11] Z. Liu, S. Guo, and Z. Zhang, "Existence and multiplicity of solutions for a class of sublinear Schrödinger-Maxwell equations," Taiwanese Journal of Mathematics, vol. 17, no. 3, pp. 857-872, 2013.

[12] D. Ruiz, "The Schrödinger-Poisson equation under the effect of a nonlinear local term," Journal of Functional Analysis, vol. 237, no. 2, pp. 655-674, 2006.

[13] Z. Wang and H.-S. Zhou, "Positive solution for a nonlinear stationary Schrödinger-Poisson system in $R^{3}$," Discrete and Continuous Dynamical Systems Series A, vol. 18, no. 4, pp. 809816, 2007.

[14] M. H. Yang and Z. Q. Han, "Existence and multiplicity results for the nonlinear Schrödinger-Poisson systems," Nonlinear
Analysis. Real World Applications, vol. 13, no. 3, pp. 1093-1101, 2012.

[15] L. Zhao and F. Zhao, "On the existence of solutions for the Schrödinger-Poisson equations," Journal of Mathematical Analysis and Applications, vol. 346, no. 1, pp. 155-169, 2008.

[16] A. Ambrosetti and D. Ruiz, "Multiple bound states for the Schrödinger-Poisson problem," Communications in Contemporary Mathematics, vol. 10, no. 3, pp. 391-404, 2008.

[17] A. Azzollini and A. Pomponio, "Ground state solutions for the nonlinear Schrödinger-Maxwell equations," Journal of Mathematical Analysis and Applications, vol. 345, no. 1, pp. 90-108, 2008.

[18] S. Chen and L. Xiao, "Existence of multiple nontrivial solutions for a strongly indefinite Schrödinger-Poisson system," Abstract and Applied Analysis, vol. 2014, Article ID 240208, 8 pages, 2014.

[19] S. J. Chen and C. L. Tang, "High energy solutions for the superlinear Schrödinger-Maxwell equations," Nonlinear Analysis. Theory, Methods \& Applications, vol. 71, no. 10, pp. 49274934, 2009.

[20] S. Cingolani, S. Secchi, and M. Squassina, "Semi-classical limit for Schrödinger equations with magnetic field and Hartree-type nonlinearities," Proceedings of the Royal Society of Edinburgh. Section A. Mathematics, vol. 140, no. 5, pp. 973-1009, 2010.

[21] M. Clapp and D. Salazar, "Positive and sign changing solutions to a nonlinear Choquard equation," Journal of Mathematical Analysis and Applications, vol. 407, no. 1, pp. 1-15, 2013.

[22] G. M. Coclite, "A multiplicity result for the nonlinear Schrödinger-Maxwell equations," Communications in Applied Analysis, vol. 7, no. 2-3, pp. 417-423, 2003.

[23] M. Willem, Minimax Theorems, Progress in Nonlinear Differential Equations and Their Applications, Birkhäuser, Boston, Mass, USA, 1996.

[24] S. Chen and C. Wang, "An infinite-dimensional linking theorem without upper semi-continuous assumption and its applications," Journal of Mathematical Analysis and Applications, vol. 420, no. 2, pp. 1552-1567, 2014.

[25] L. C. Evans, Partial Differential Equations, vol. 19 of Graduate Studies in Mathematics, American Mathematical Society, Providence, RI, USA, 1998.

[26] E. H. Lieb, "Sharp constants in the Hardy-Littlewood-Sobolev and related inequalities," Annals of Mathematics, vol. 118, no. 2, pp. 349-374, 1983.

[27] D. Ruiz, "On the Schrödinger-Poisson-Slater System: behavior of minimizers, radial and nonradial cases," Archive for Rational Mechanics and Analysis, vol. 198, no. 1, pp. 349-368, 2010.

[28] D. Gilbarg and N. S. Trudinger, Elliptic Partial Differential Equations of Second Order, Classics in Mathematics, Springer, Berlin, Germany, 2001. 


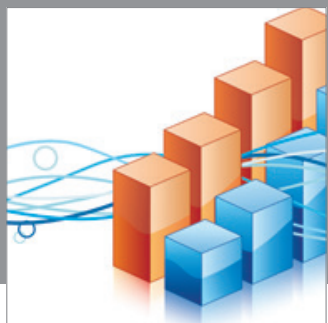

Advances in

Operations Research

mansans

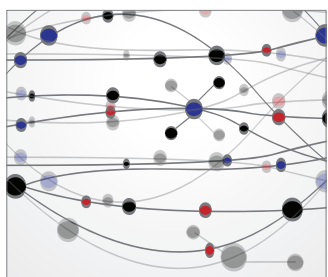

The Scientific World Journal
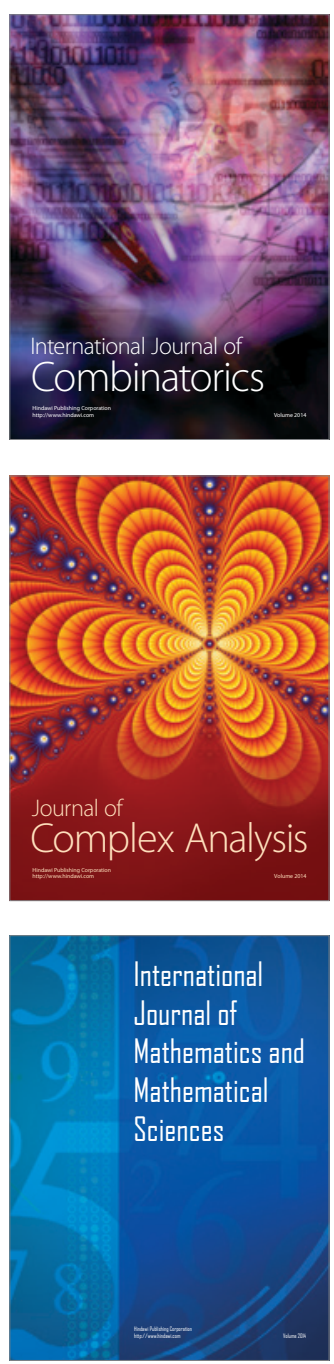
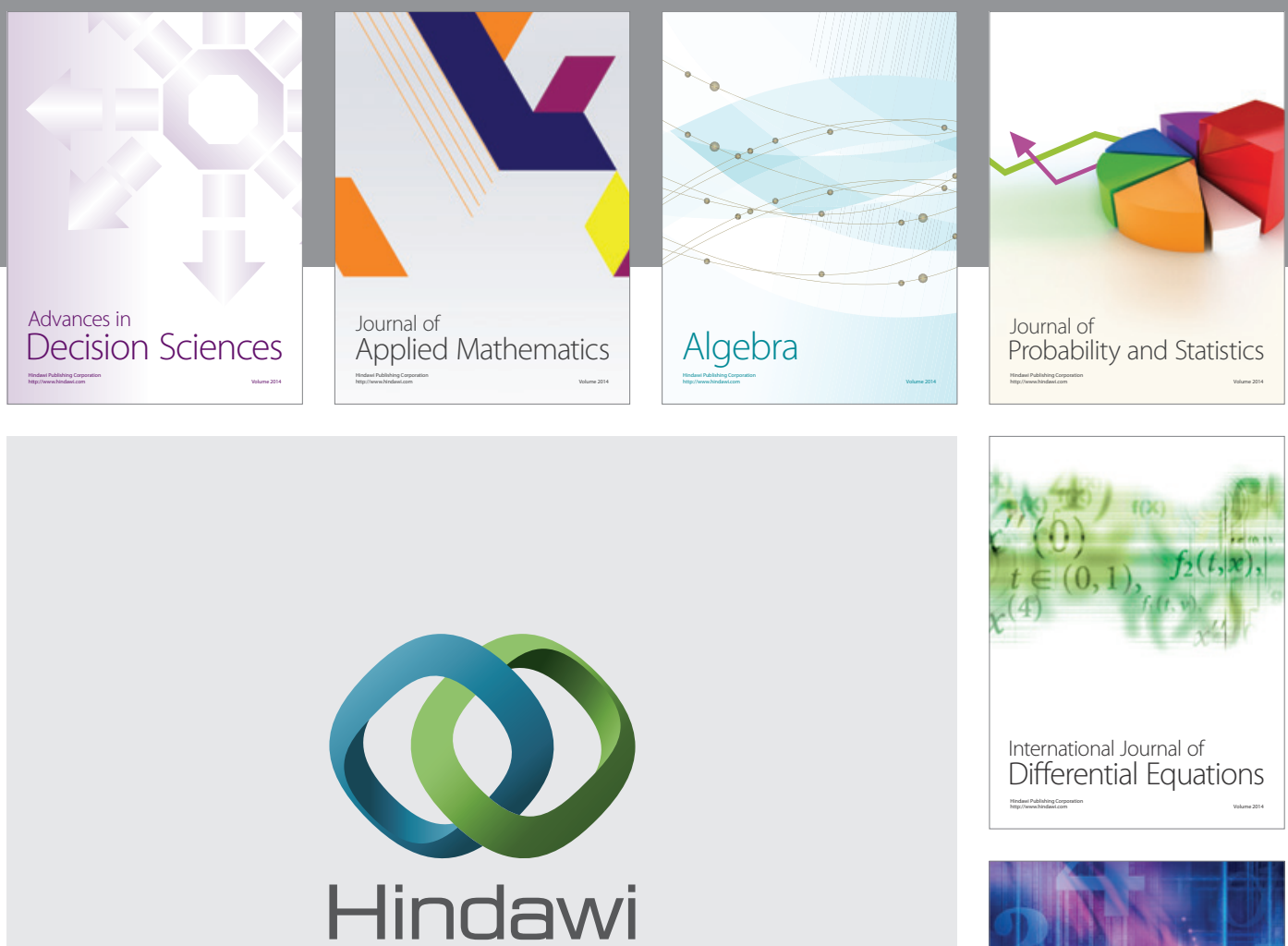

Submit your manuscripts at http://www.hindawi.com
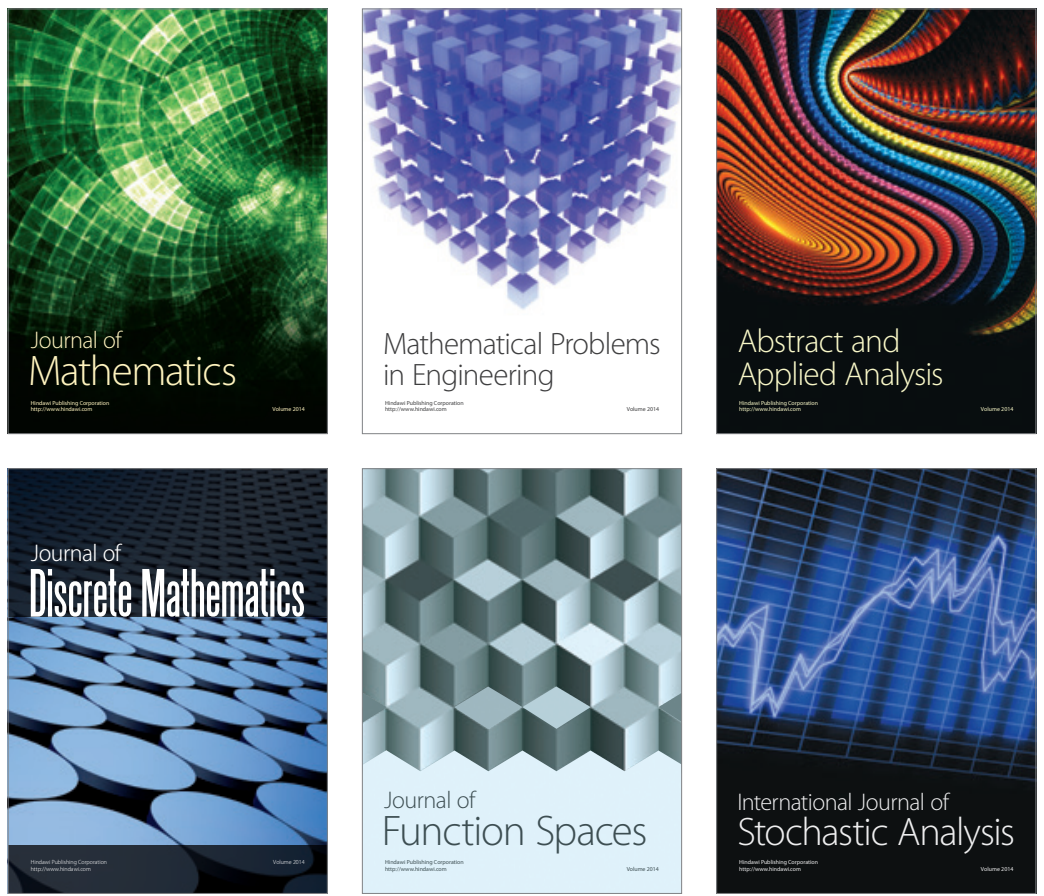

Journal of

Function Spaces

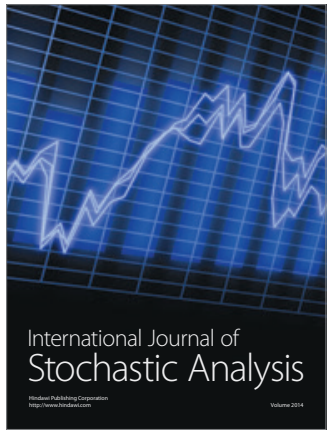

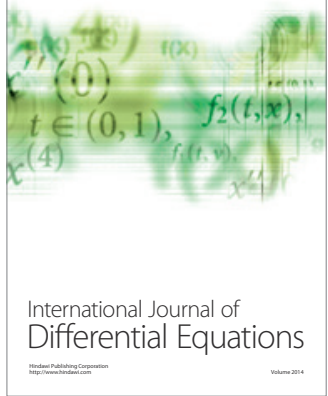
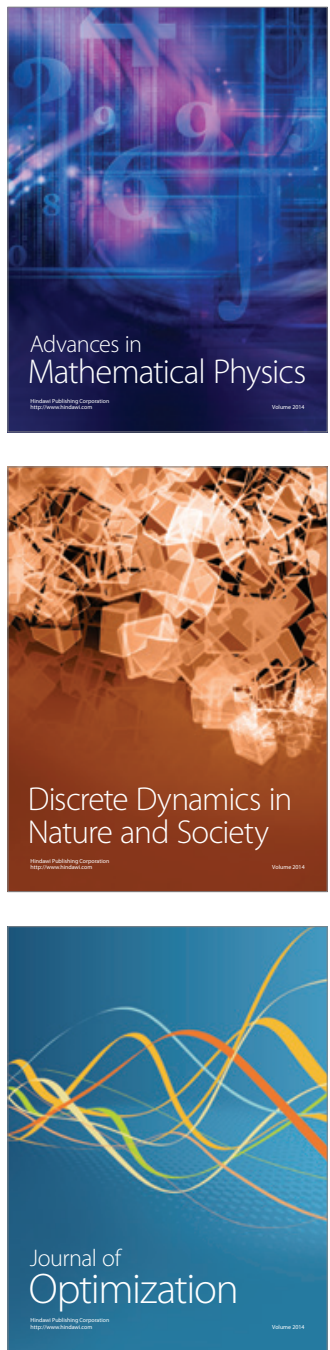